\title{
BIBO Stability Analysis for Delay Switched Systems with Nonlinear Perturbation
}

\author{
Jincheng Wei, ${ }^{1}$ Peng Shi, ${ }^{2,3}$ Hamid Reza Karimi, ${ }^{4}$ and Bo Wang ${ }^{1}$ \\ ${ }^{1}$ School of Electrical and Information Engineering, Xihua University, Chengdu 610096, China \\ ${ }^{2}$ College of Engineering and Science, Victoria University, Melbourne, VIC 8001, Australia \\ ${ }^{3}$ School of Electrical and Electronic Engineering, The University of Adelaide, Adelaide, SA 5005, Australia \\ ${ }^{4}$ Department of Engineering, Faculty of Engineering and Science, University of Agder, 4898 Grimstad, Norway
}

Correspondence should be addressed to Hamid Reza Karimi; hamid.r.karimi@uia.no

Received 16 February 2013; Revised 15 April 2013; Accepted 17 April 2013

Academic Editor: Hongli Dong

Copyright (c) 2013 Jincheng Wei et al. This is an open access article distributed under the Creative Commons Attribution License, which permits unrestricted use, distribution, and reproduction in any medium, provided the original work is properly cited.

\begin{abstract}
The problem of bounded-input bounded-output (BIBO) stability is investigated for a class of delay switched systems with mixed time-varying discrete and constant neutral delays and nonlinear perturbation. Based on the Lyapunov-Krasovskii functional theory, new BIBO stabilization criteria are established in terms of delay-dependent linear matrix inequalities. The numerical simulation is carried out to demonstrate the effectiveness of the results obtained in the paper.
\end{abstract}

\section{Introduction}

Time delay is a source of instability and poor performance and appears in many dynamic systems, for example, biological systems, chemical systems, metallurgical processing systems, nuclear reactor systems, and electrical networks [1]. Since the existence of time delays may lead to oscillation, divergence, or instability, considerable effort has been devoted to this area. As an important system performance index, BIBO stability means that any bounded input yields a bounded output and can be considered in many aspects, such as the free system dynamics, the basic single or double loop modulators, and the issues connected with bilinear input/output maps. Consequently, bounded-input boundedoutput (BIBO) stability analysis of dynamical systems has attracted many scholars' attention. For instance, in [2], BIBO stability criterion is derived for a three-dimensional fuzzy two-term control system, in [3], the problem on BIBO stabilization for a system with nonlinear perturbations is studied by discussing the existence of the positive definite solution to an auxiliary algebraic Riccati matrix equation, in [4], based on linear matrix inequality techniques, the stabilization criterion for uncertain time-delay system is presented to guarantee that bounded input can lead to bounded output, and in [5], BIBO stability for feedback control systems with time delay is studied through investigating the boundedness of the solutions for a class of nonlinear Volterra integral equations.

Recently, switched system becomes a research hotspot. Its motivation comes from the fact that many practical systems are inherently multimodal and the fact that some of intelligent control methods are based on the idea of switching between different controllers. Up till now, many investigations about stability of multiform switched systems have been carried out; see, for instance, [6-19] and references therein. Hence, it is our intention in this paper to tackle such an important yet challenging problem for BIBO stability analysis of delay switched systems. In addition, perturbations [20-26] and time delays [27-29] exist in many kinds of systems, and this makes the practical control problem complicated and has received much attention from 
scholars. Hence, in this paper, the BIBO stability for delay switched system with mixed time-varying discrete and constant neutral delays and nonlinear perturbation is concerned, and some original BIBO stability criteria are established in terms of linear matrix inequalities (LMIs). Finally, some simulation results are given to illustrate the effectiveness of our results. The main contributions of the paper are of two folds: (1) a delay-dependent technique is applied successfully into the analysis results process; (2) a LyapunovKrasovskii functional is constructed to derive a new form of the bounded real lemma (BRL) for the system under consideration.

The remainder of this paper is organized as follows. The model under consideration and some preliminaries are provided in Section 2. Section 3 presents the results on stability analysis. Section 4 gives an illustrative example. At last we conclude the paper in Section 5.

Notations used in this paper are fairly standard. Let $R^{n}$ be the $n$-dimensional Euclidean space, $R^{n \times m}$ represents the set of $n \times m$ real matrices, the symbol ${ }^{*}$ denotes the elements below the main diagonal of a symmetric block matrix, $A>0$ means that $A$ is a real symmetric positive definitive matrix, and $I$ denotes the identity matrix with appropriate dimensions. $\operatorname{diag}\{\cdots\}$ denotes the diagonal matrix. $E\{\cdot\}$ refers to the expectation operator with respect to some probability measure $P$. $\|\cdot\|$ refers to the Euclidean vector norm or the induced matrix 2-norm. The superscript $T$ stands for matrix transposition. $L_{n, h}=L\left([-h, 0], R^{n}\right)$ denotes the Banach space of continuous functions mapping the interval $[-h, 0]$ into $R^{n}$ with the topology of uniform convergence.

\section{Model Description and Preliminaries}

First, consider the following delay switched system with nonlinear perturbation:

$$
\begin{gathered}
\dot{x}(t)-C_{\sigma(t)} \dot{x}(t-d)=A_{\sigma(t)} x(t)+B_{\sigma(t)} x(t-\tau(t)) \\
+f_{\sigma(t)}(t, x(t))+H_{\sigma(t)} u(t), \\
u(t)=L_{\sigma(t)} x(t)+r(t), \\
Y(t)=J x(t), \\
x\left(t_{0}+\theta\right)=\varphi(\theta), \quad \theta \in\left[\begin{array}{ll}
-h & 0
\end{array}\right],
\end{gathered}
$$

where $x(t) \in R^{n}$ is the state vector, $d$ is the neutral delay, $0 \leq \tau(t) \leq h$ is the time-varying discrete delay, $\varphi(\theta) \in L_{n, h}$ is the initial condition, $\sigma(t):[0,+\infty) \rightarrow M=\{1,2, \ldots, m\}$ is the switching signal, $u(t) \in R^{l}$ is the control input, $Y(t) \in$ $R^{m}$ is the system output, $r(t) \in R^{l}$ is the reference input, and $f(t) \in R^{n}$ is the nonlinear time-varying perturbation, which satisfies $\|f(t, x(t))\| \leq \beta\|x(t)\|$, where $\beta$ is a positive scalar.
Model (1) can be represented as follows:

$$
\begin{gathered}
\dot{x}(t)=y(t), \\
y(t)-C_{\sigma(t)} y(t-d)=A_{\sigma(t)} x(t)+B_{\sigma(t)} x(t-\tau(t)) \\
+f_{\sigma(t)}(t)+H_{\sigma(t)} u(t) .
\end{gathered}
$$

In this paper, the following well-known lemmas and definitions are needed.

Lemma 1 (see [30]). For any constant matrices $E, G$, and $F$ with appropriate dimensions with $F^{T} F \leq k I$, then

$$
2 x^{T} E F G y \leq c x E E^{T} x+\frac{k}{c} y^{T} G^{T} G y
$$

where $x \in R^{n}$ and $y \in R^{n}$ and $c$ and $k$ are positive scalars.

Lemma 2 (see [31]). For any positive definite matrix $\Phi \in R^{n \times n}$, a positive scalar $\gamma$, and the vector function $w:[0, \gamma] \rightarrow R^{n}$ such that the integrations concerned are well defined, then

$$
\left(\int_{0}^{\gamma} w(s) d s\right)^{T} \Phi\left(\int_{0}^{\gamma} w(s) d s\right) \leq \gamma \int_{0}^{\gamma} w^{T}(s) \Phi w(s) d s
$$

Definition 3 (see [32]). A real-valued vector $r(t) \in L_{\infty}^{n}$, if $\|r\|_{\infty}=\sup _{t_{0} \leq t<\infty}\|r(t)\|<+\infty$.

Definition 4 (see [32]). The control system with reference input $r(t)$ is BIBO stable, if there exist some positive constants $\theta_{1}, \theta_{2}$, satisfying

$$
\|Y(t)\| \leq \theta_{1}\|r(t)\|_{\infty}+\theta_{2}
$$

for any reference input $r(t) \in L_{\infty}^{n}$.

Assumption 5. We assume that for system (1) there exist Hurwitz linear convex combinations of $A_{i}$; that is,

$$
\begin{aligned}
& \gamma_{\alpha_{1}, \alpha_{2}, \ldots, \alpha_{m}}\left(A_{1}, A_{2}, \ldots, A_{m}\right) \\
& =\left\{\alpha_{1} A_{1}+\alpha_{2} A_{2}+\cdots+\alpha_{m} A_{m}: \alpha_{1}, \alpha_{2}, \ldots \alpha_{m} \in[0,1],\right. \\
& \left.\alpha_{1}+\alpha_{2}+\cdots+\alpha_{m}=1\right\} .
\end{aligned}
$$

\section{Main Results}

In this section, we will establish some BIBO stability criteria using Lyapunov-Krasovskii functional theory and linear matrix inequalities.

Theorem 6. For given positive scalars $h$ and $k$, switched system (1) is BIBO stable, if there exist $A \in$ $\gamma_{\alpha_{1}, \alpha_{2}, \ldots, \alpha_{m}}\left(A_{1}, A_{2}, \ldots, A_{m}\right), B \in \gamma_{\alpha_{1}, \alpha_{2}, \ldots, \alpha_{m}}\left(B_{1}, B_{2}, \ldots, B_{m}\right)$, $C \in \gamma_{\alpha_{1}, \alpha_{2}, \ldots, \alpha_{m}}\left(C_{1}, C_{2}, \ldots, C_{m}\right), f \in \gamma_{\alpha_{1}, \alpha_{2}, \ldots, \alpha_{m}}\left(f_{1}, f_{2}, \ldots, f_{m}\right)$, $H \in \gamma_{\alpha_{1}, \alpha_{2}, \ldots, \alpha_{m}}\left(H_{1}, H_{2}, \ldots, H_{m}\right), L \stackrel{\epsilon}{\epsilon} \gamma_{\alpha_{1}, \alpha_{2}, \ldots, \alpha_{m}}\left(L_{1}\right.$, $\left.L_{2}, \ldots, L_{m}\right)$, positive scalars $\varepsilon, \sigma$, matrices $P_{2}, P_{3}, U, V, W$, 
and symmetric positive definite matrices $P, R, M, S, Q$, satisfying

$$
\begin{gathered}
\Sigma+\Xi+\Xi^{T}+h e^{k h} W<0, \\
{\left[\begin{array}{cc}
W & U \\
* & S-R_{22}
\end{array}\right]>0,} \\
{\left[\begin{array}{cc}
W & V \\
* & S
\end{array}\right]>0,}
\end{gathered}
$$

where

$$
\begin{aligned}
& \Sigma=\left[\begin{array}{ccccccc}
\Sigma_{1,1} & \Sigma_{1,2} & \Sigma_{1,3} & \Sigma_{1,4} & \Sigma_{1,5} & \Sigma_{1,6} & \Sigma_{1,7} \\
* & \Sigma_{2,2} & \Sigma_{2,3} & \Sigma_{2,4} & \Sigma_{2,5} & \Sigma_{2,6} & \Sigma_{2,7} \\
* & * & \Sigma_{3,3} & 0 & 0 & 0 & 0 \\
* & * & * & \Sigma_{4,4} & 0 & 0 & 0 \\
* & * & * & * & \Sigma_{5,5} & 0 & 0 \\
* & * & * & * & * & \Sigma_{6,6} & 0 \\
* & * & * & * & * & * & \Sigma_{7,7}
\end{array}\right] \\
& \Sigma_{1,1}=P_{2} A+A^{T} P_{2}^{T}+P_{2} H L+L^{T} H^{T} P_{2}^{T} \\
& +Q+\varepsilon \beta^{2}+k P+h^{2} e^{k h} N, \\
& \Sigma_{1,2}=P-P_{2}+A^{T} P_{3}^{T}+L^{T} H^{T} P_{3}^{T}, \\
& \Sigma_{1,3}=P_{2} B+R_{12}^{T} \text {, } \\
& \Sigma_{2,2}=h e^{k h} S-P_{3}-P_{3}^{T}+M \text {, } \\
& \Sigma_{2,3}=P_{3} B \text {, } \\
& \Sigma_{3,3}=h R_{11}-R_{12}-R_{12}^{T} \text {, } \\
& \Sigma_{1,4}=P_{2} C \text {, } \\
& \Sigma_{2,4}=P_{3} C \text {, } \\
& \Sigma_{4,4}=-e^{-k d} M \text {, } \\
& \Sigma_{1,5}=P_{2} \text {, } \\
& \Sigma_{2,5}=P_{3} \text {, } \\
& \Sigma_{5,5}=-\varepsilon I \text {, } \\
& \Sigma_{1,6}=0 \text {, } \\
& \Sigma_{2,6}=0 \text {, } \\
& \Sigma_{6,6}=-e^{-k h} Q \text {, } \\
& \Sigma_{1,7}=P_{2} H \text {, } \\
& \Sigma_{2,7}=P_{3} H \text {, } \\
& \Sigma_{7,7}=-\sigma I \text {, } \\
& \Xi=\left[\begin{array}{lllllll}
U & 0 & -U+V & 0 & 0 & -V & 0
\end{array}\right] .
\end{aligned}
$$

Proof. Since

$$
\begin{aligned}
& A \in \gamma_{\alpha_{1}, \alpha_{2}, \ldots, \alpha_{m}}\left(A_{1}, A_{2}, \ldots, A_{m}\right) \text {, } \\
& B \in \gamma_{\alpha_{1}, \alpha_{2}, \ldots, \alpha_{m}}\left(B_{1}, B_{2}, \ldots, B_{m}\right), \\
& C \in \gamma_{\alpha_{1}, \alpha_{2}, \ldots, \alpha_{m}}\left(C_{1}, C_{2}, \ldots, C_{m}\right) \\
& L \in \gamma_{\alpha_{1}, \alpha_{2}, \ldots, \alpha_{m}}\left(L_{1}, L_{2}, \ldots, L_{m}\right) \text {, } \\
& H \in \gamma_{\alpha_{1}, \alpha_{2}, \ldots, \alpha_{m}}\left(H_{1}, H_{2}, \ldots, H_{m}\right) \text {, } \\
& f \in \gamma_{\alpha_{1}, \alpha_{2}, \ldots, \alpha_{m}}\left(f_{1}, f_{2}, \ldots, f_{m}\right), \\
& \sum_{i=1}^{m} \alpha_{i}=1, \quad A=\sum_{i=1}^{m} \alpha_{i} A_{i}, \quad B=\sum_{i=1}^{m} \alpha_{i} B_{i}, \\
& C=\sum_{i=1}^{m} \alpha_{i} C_{i}, \quad L=\sum_{i=1}^{m} \alpha_{i} L_{i}, \\
& H=\sum_{i=1}^{m} \alpha_{i} H_{i}, \quad f=\sum_{i=1}^{m} \alpha_{i} f_{i} .
\end{aligned}
$$

From (7), we can obtain

$$
\sum_{i=1}^{m} \alpha_{i}\left(\Sigma_{i}+\Xi+\Xi^{T}+h e^{k h} W\right)<0,
$$

where

$$
\Sigma_{i}=\left[\begin{array}{ccccccc}
\Sigma_{i, 1,1} & \Sigma_{i, 1,2} & \Sigma_{i, 1,3} & \Sigma_{i, 1,4} & \Sigma_{i, 1,5} & \Sigma_{i, 1,6} & \Sigma_{i, 1,7} \\
* & \Sigma_{i, 2,2} & \Sigma_{i, 2,3} & \Sigma_{i, 2,4} & \Sigma_{i, 2,5} & \Sigma_{i, 2,6} & \Sigma_{i, 2,7} \\
* & * & \Sigma_{i, 3,3} & 0 & 0 & 0 & 0 \\
* & * & * & \Sigma_{i, 4,4} & 0 & 0 & 0 \\
* & * & * & * & \Sigma_{i, 5,5} & 0 & 0 \\
* & * & * & * & * & \Sigma_{i, 6,6} & 0 \\
* & * & * & * & * & * & \Sigma_{i, 7,7}
\end{array}\right],
$$




$$
\begin{aligned}
\Sigma_{i, 1,1}= & P_{2} A_{i}+A_{i}^{T} P_{2}^{T}+P_{2} H_{i} L_{i} \\
& +L_{i}^{T} H_{i}^{T} P_{2}^{T}+Q+\varepsilon \beta^{2}+k P+h^{2} e^{k h} N, \\
\Sigma_{i, 1,2}= & P-P_{2}+A_{i}^{T} P_{3}^{T}+L_{i}^{T} H_{i}^{T} P_{3}^{T}, \\
\Sigma_{i, 1,3}= & P_{2} B_{i}+R_{12}^{T}, \\
\Sigma_{i, 2,2}= & h e^{k h} S-P_{3}-P_{3}^{T}+M, \\
\Sigma_{i, 2,3}= & P_{3} B_{i}, \\
\Sigma_{i, 3,3}= & h R_{11}-R_{12}-R_{12}^{T}, \\
\Sigma_{i, 1,4}= & P_{2} C_{i}, \\
\Sigma_{i, 2,4}= & P_{3} C_{i}, \\
\sum_{i, 4,4}= & -e^{k d} M, \\
\Sigma_{i, 1,5}= & P_{2}, \\
\Sigma_{i, 2,5}= & P_{3}, \\
\Sigma_{i, 5,5}= & -\varepsilon I, \\
\Sigma_{i, 1,6}= & 0, \\
\sum_{i, 2,6}= & 0, \\
\Sigma_{i, 6,6}= & -e^{-k h} Q, \\
\Sigma_{i, 1,7}= & P_{2} H_{i}, \\
\sum_{i, 2,7}= & P_{3} H_{i}, \\
\Sigma_{i, 7,7}= & -\sigma I .
\end{aligned}
$$

Let

$$
\begin{aligned}
\Omega_{i}=\left\{q^{T} \mid q^{T}\left(\Sigma_{i}+\Xi+\Xi^{T}+h W\right) q<0,\right. \\
\left.q(t)=\left[q_{1}^{T}, \ldots, q_{i}^{T}, \ldots, q_{7}^{T}\right]^{T}, q_{i} \in R^{n}\right\} .
\end{aligned}
$$

We obtain

$$
\bigcup_{i=1}^{m} \Omega_{i}=\frac{R^{7 n}}{\{0\}}
$$

\section{Construct a set as}

$$
\begin{aligned}
& \widetilde{\Omega}_{1}=\Omega_{1}, \\
& \widetilde{\Omega}_{2}=\Omega_{2}-\widetilde{\Omega}_{1}, \ldots, \\
& \widetilde{\Omega}_{i}=\Omega_{i}-\bigcup_{j=1}^{i-1} \widetilde{\Omega}_{j}, \ldots, \\
& \widetilde{\Omega}_{m}=\Omega_{m}-\bigcup_{j=1}^{m-1} \widetilde{\Omega}_{j} .
\end{aligned}
$$

We get

$$
\bigcup_{i=1}^{m} \widetilde{\Omega}_{i}=\frac{R^{6 n}}{\{0\}}, \quad \widetilde{\Omega}_{i} \cap \widetilde{\Omega}_{j}=\phi, \quad i \neq j .
$$

Construct the switching rule (SR): $\sigma=i$, for all $q \epsilon$ $\widetilde{\Omega}_{i}, i=1, \ldots, m$. The $i$ th subsystem is activated when $q \in$ $\widetilde{\Omega}_{i}, i=1, \ldots, m$. Choose the following Lyapunov-Krasovskii functional candidate:

$$
\begin{aligned}
V(t)= & V_{1}(t)+V_{2}(t)+V_{3}(t) \\
& +V_{4}(t)+V_{5}(t)+V_{6}(t),
\end{aligned}
$$

with

$$
\begin{aligned}
& V_{1}(t)=\left(x^{T}(t) y^{T}(t)\right)\left[\begin{array}{ll}
I & 0 \\
0 & 0
\end{array}\right]\left[\begin{array}{cc}
P & 0 \\
P_{2}^{T} & P_{3}^{T}
\end{array}\right]\left(x^{T}(t) y^{T}(t)\right)^{T}, \\
& V_{2}(t)=\int_{-h}^{0} \int_{t+\beta}^{t} y^{T}(\alpha) e^{k(\alpha-t+h)} S y(\alpha) d \alpha d \beta, \\
& V_{3}(t)=\int_{-h}^{t} \int_{\beta-\tau(\beta)}^{\beta} \eta^{T} e^{k(\beta-t)} R \eta d \alpha d \beta, \\
& V_{4}(t)=\int_{t-d}^{t} y^{T}(s) e^{k(s-t)} M y(s) d s, \\
& V_{5}(t)=\int_{-h}^{0} \int_{t+\beta}^{t} \xi^{T}(\alpha) e^{k(\alpha-t+h)} W \xi(\alpha) d \alpha d \beta, \\
& V_{6}(t)=\int_{t-h}^{t} x^{T}(s) e^{k(s-t)} Q x(s) d s,
\end{aligned}
$$

where

$$
\begin{aligned}
& \eta=\left[\begin{array}{lll}
x(\beta-\tau(\beta)) & y(\alpha)
\end{array}\right]^{T}, \\
& \xi=\left[\begin{array}{lllllll}
x^{T}(t) & y^{T}(t) & x^{T}(t-\tau(t)) & y^{T}(t-d) & f^{T}(t) & x(t-h) & r(t)
\end{array}\right]^{T} .
\end{aligned}
$$

The derivative of $V(t)$ along the trajectory of the $i$ th subsystem is given by

$$
\begin{aligned}
\dot{V}(t)= & \dot{V}_{1}(t)+\dot{V}_{2}(t)+\dot{V}_{3}(t) \\
& +\dot{V}_{4}(t)+\dot{V}_{5}(t)+\dot{V}_{6}(t),
\end{aligned}
$$


where

$$
\begin{aligned}
& \dot{V}_{1}(t)=2\left[x^{T}(t) y^{T}(t)\right]\left[\begin{array}{ll}
P & P_{2} \\
0 & P_{3}
\end{array}\right]\left[\begin{array}{c}
y(t) \\
0
\end{array}\right] \\
& =2 x^{T}(t) P y(t)+2\left(x^{T}(t) P_{2}+y^{T}(t) P_{3}\right) \\
& \times\left(-y(t)+\left(A_{i}+H_{i} L_{i}\right) x(t)\right. \\
& +B_{i} x(t-\tau(t))+C_{i} y(t-d) \\
& \left.+f_{i}(t)+H_{i} r(t)\right), \\
& \dot{V}_{2}(t)=h y^{T}(t) e^{k h} S y(t) \\
& -\int_{t-\tau(t)}^{t} y^{T}(s) e^{k(s-t+h)} S y(s) d s \\
& -\int_{t-h}^{t-\tau(t)} y^{T}(s) e^{k(s-t+h)} S y(s) d s-k V_{2}(t) \\
& \leq h y^{T}(t) e^{k h} S y(t) \\
& -\int_{t-\tau(t)}^{t} y^{T}(s) S y(s) d s \\
& -\int_{t-h}^{t-\tau(t)} y^{T}(s) S y(s) d s-k V_{2}(t), \\
& \dot{V}_{3}(t)=\tau(t) x^{T}(t-\tau(t)) R_{11} x(t-\tau(t)) \\
& +2 x^{T}(t-\tau(t)) R_{12} x(t) \\
& -2 x^{T}(t-\tau(t)) R_{12} x(t-\tau(t)) \\
& +\int_{t-\tau(t)}^{t} y(s) R_{22} y(s) d s-k V_{3}(t) \\
& \leq h x^{T}(t-\tau(t)) R_{11} x(t-\tau(t)) \\
& +2 x^{T}(t) R_{12}^{T} x(t-\tau(t)) \\
& -2 x^{T}(t-\tau(t)) R_{12} x(t-\tau(t)) \\
& +\int_{t-\tau(t)}^{t} y(s) R_{22} y(s) d s-k V_{3}(t), \\
& \dot{V}_{4}(t)=y^{T}(t) M y(t)-y^{T}(t-d) e^{-k d} M y(t-d)-k V_{4}(t) \text {, } \\
& \dot{V}_{5}(t)=h \xi^{T}(t) e^{k h} W \xi(t)-\int_{t-\tau(t)}^{t} \xi^{T}(s) e^{k(s-t+h)} W \xi(s) d s \\
& -\int_{t-h}^{t-\tau(t)} \xi^{T}(s) e^{k(s-t+h)} W \xi(s) d s-k V_{5}(t) \\
& \leq h \xi^{T}(t) e^{k h} W \xi(t)-\int_{t-\tau(t)}^{t} \xi^{T}(s) W \xi(s) d s \\
& -\int_{t-h}^{t-\tau(t)} \xi^{T}(s) W \xi(s) d s-k V_{5}(t),
\end{aligned}
$$

$$
\dot{V}_{6}(t)=x^{T}(t) Q x(t)-x^{T}(t-h) e^{-k h} Q x(t-h)-k V_{6}(t) .
$$

According to Leibniz-Newton formula, we have

$$
\begin{gathered}
2 \xi^{T} U\left[x(t)-x(t-\tau(t))-\int_{t-\tau(t)}^{t} y^{T}(s) d s\right]=0, \\
2 \xi^{T} V\left[x(t-\tau(t))-x(t-h)-\int_{t-h}^{t-\tau(t)} y^{T}(s) d s\right]=0 .
\end{gathered}
$$

From the obtained derivative terms in (21)-(26) and adding the left-hand side of (27) into (20), we obtain the following result:

$$
\begin{aligned}
\dot{V}(t) \leq & \xi^{T}\left(\Sigma_{i}+\Omega+\Omega^{T}+h e^{k h} W\right) \xi \\
& -\int_{t-\tau(t)}^{t} \zeta^{T} \Phi_{1} \zeta d s-\int_{t-h}^{t-\tau(t)} \zeta^{T} \Phi_{2} \zeta d s \\
& -k V(t)+\sigma\|r(t)\|_{\infty}^{2},
\end{aligned}
$$

where

$$
\begin{gathered}
\zeta=\left[\begin{array}{ll}
\xi^{T} & y^{T}(s)
\end{array}\right]^{T}, \\
\Phi_{1}=\left[\begin{array}{cc}
W & U \\
* & S-R_{22}
\end{array}\right], \quad \Phi_{2}=\left[\begin{array}{cc}
W & V \\
* & S
\end{array}\right] .
\end{gathered}
$$

When $\xi \in \bigcup_{i=1}^{m} \widetilde{\Omega}_{i}, i=1, \ldots, m$, we can obtain

$$
\begin{gathered}
\dot{V}(t) \leq \sum_{i=1}^{m} a_{i}\left(\xi^{T}\left(\Sigma_{i}+\Omega+\Omega^{T}+h e^{k h} W\right) \xi\right. \\
\left.\quad-\int_{t-\tau(t)}^{t} q^{T} \Phi_{1} q d s-\int_{t-h}^{t-\tau(t)} q^{T} \Phi_{2} q d s\right) \\
=\xi^{T}\left(\Sigma+\Xi+\Xi^{T}+h e^{k h} W\right) \xi \\
-\int_{t-\tau(t)}^{t} q^{T} \Phi_{1} q d s-\int_{t-h}^{t-\tau(t)} q^{T} \Phi_{2} q d s .
\end{gathered}
$$

According to (7), we have

$$
\dot{V}(t) \leq-k V(t)+\sigma\|r(t)\|_{\infty}^{2} .
$$

Hence,

$$
\left(V(t) e^{k t}\right)^{\prime} \leq(\dot{V}(t)+k V(t)) e^{k t} \leq \sigma\|r(t)\|_{\infty}^{2} e^{k t} .
$$

Integrating the previous inequality from $t_{0}$ to $t$ yields

$$
V(t) e^{k t} \leq V\left(t_{0}\right) e^{k t_{0}}+\sigma\|r(t)\|_{\infty}^{2} \int_{t_{0}}^{t} e^{k s} d s .
$$

Then, we have

$$
\begin{aligned}
\lambda_{\min }(P)\|x(t)\|^{2} \leq & V(t) \leq V\left(t_{0}\right) e^{-k\left(t-t_{0}\right)} \\
& +\sigma\|r(t)\|_{\infty}^{2} \int_{t_{0}}^{t} e^{-k(t-s)} d s \\
\leq & V\left(t_{0}\right) e^{-k\left(t-t_{0}\right)}+\frac{\sigma\|r(t)\|_{\infty}^{2}}{k} .
\end{aligned}
$$


Define

$$
\psi=\max \left\{\sup _{h \leq \theta \leq 0}\left\|\varphi\left(t_{0}+\theta\right)\right\|, \sup _{h \leq \theta \leq 0}\left\|\varphi^{\prime}\left(t_{0}+\theta\right)\right\|\right\} .
$$

According to (17), we have

$$
\begin{aligned}
V\left(t_{0}\right) \leq[ & \lambda_{\max }(P)+h^{2} e^{k h} \lambda_{\max }(S)+h^{2} \lambda_{\max }(R) \\
& \left.+h^{2} e^{k h} \lambda_{\max }(W)+h \lambda_{\max }(Q)+h \lambda_{\max }(M)\right] \psi^{2} .
\end{aligned}
$$

Hence, the following inequality can be concluded:

$$
\begin{aligned}
\|x(t)\|^{2} & \leq \frac{a}{\lambda_{\text {min }}(P)} \psi^{2}+\frac{\sigma}{k \lambda_{\min }(P)}\|r(t)\|_{\infty}^{2} \\
& \leq\left(\sqrt{\frac{a}{\lambda_{\text {min }}(P)}} \psi+\sqrt{\frac{\sigma}{k \lambda_{\text {min }}(P)}}\|r(t)\|_{\infty}\right)^{2},
\end{aligned}
$$

where

$$
\begin{aligned}
a= & \lambda_{\max }(P)+h^{2} e^{k h} \lambda_{\max }(S)+h^{2} \lambda_{\max }(R) \\
& +h^{2} e^{k h} \lambda_{\max }(W)+h \lambda_{\max }(Q)+h \lambda_{\max }(M) .
\end{aligned}
$$

Then,

$$
\|Y\| \leq\|J\|\|x\| \leq \theta_{1}+\theta_{2}\|r(t)\|_{\infty},
$$

with

$$
\theta_{1}=\|J\| \sqrt{\frac{a}{\lambda_{\min }(P)}} \psi, \quad \theta_{2}=\|J\| \sqrt{\frac{\sigma}{k \lambda_{\text {min }}(P)}} .
$$

Therefore, switched system (1) is BIBO stable. This completes the proof.

\section{Simulation Results}

As an example, let us consider system (1) with the following parameters:

$$
\begin{array}{rlr}
A_{1}=\left[\begin{array}{cc}
-1.5 & 0.5 \\
0 & -1.5
\end{array}\right], & B_{1}=\left[\begin{array}{cc}
-0.2 & 0.3 \\
0 & -0.4
\end{array}\right], \\
C_{1}=\left[\begin{array}{cc}
0.3 & 0.2 \\
0 & -0.2
\end{array}\right], & H_{1}=\left[\begin{array}{cc}
0.4 & 0 \\
0 & 0.4
\end{array}\right], \\
L_{1}=\left[\begin{array}{cc}
0.2 & 0 \\
0 & 0.2
\end{array}\right], & A_{2}=\left[\begin{array}{cc}
-1 & 0.5 \\
0.5 & -2
\end{array}\right], \\
B_{2}=\left[\begin{array}{cc}
-0.4 & 0 \\
0.3 & -0.4
\end{array}\right], & C_{2}=\left[\begin{array}{cc}
-0.4 & 0.3 \\
0 & -0.3
\end{array}\right], \\
H_{2}=\left[\begin{array}{cc}
-0.3 & 0 \\
0 & -0.3
\end{array}\right], & L_{2}=\left[\begin{array}{cc}
0.3 & 0 \\
0 & 0.3
\end{array}\right], \\
J=\left[\begin{array}{ll}
1 & 0 \\
0 & 1
\end{array}\right], & d=1.5 .
\end{array}
$$

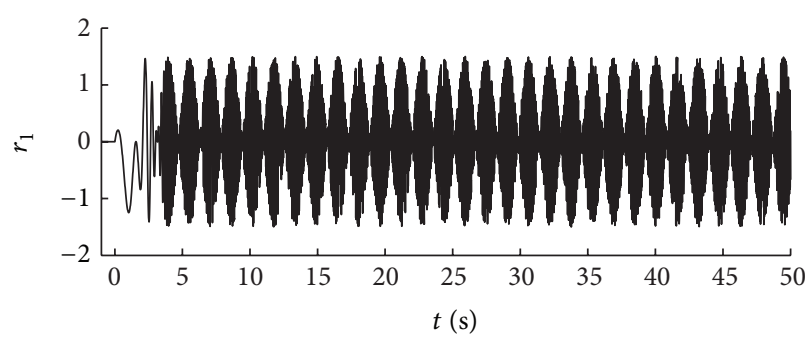

(a)

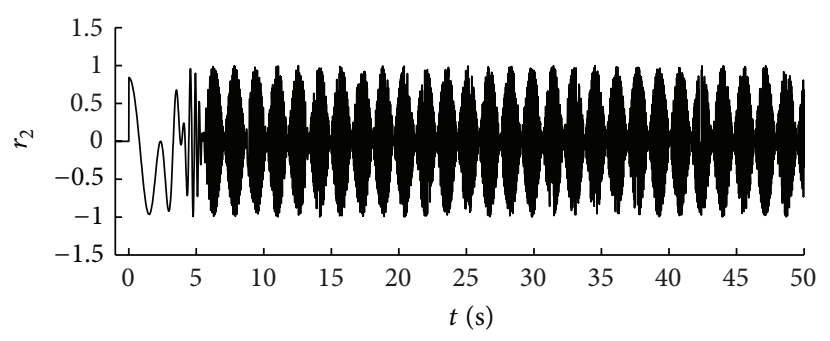

(b)

FIGURE 1: Time response of the reference input variable $r(t)$.

Remark 7. When the BIBO parameter $k$ and the constant parameter $\beta$ are given, the upper bound of time delay $h$ of system (1) can be determined by solving the following optimization problem:

$\max h$

when (7) is satisfied, $k$ and $\beta$ are fixed.

Now we consider the influence of parameters $k$ and $\beta$ on the maximal allowable delay in Tables 1 and 2.

Remark 8. From Tables 1 and 2, it can be seen that the maximal allowable delay decreases with the rise of the parameter $k$ and increases as the parameter $\beta$ is reduced.

Then, we carry out some numerical simulation to verify the proposed methodology. The numerical simulation is with initial value $\varphi(\theta)=[-1.5 ; 1]^{T}, t \in(-1.5,0)$, and following parameters

$$
\begin{gathered}
\tau(t)=1.1+0.4 \cos ^{2}(5 t), \\
f(t, x(t))=\frac{\beta[|x(t)+1|+|x(t)-1|]}{2}, \\
r(t)=\left[1.5 \sin (2 t) \cos \left(e^{t}\right), \cos (2 t) \sin \left(\frac{e^{t}}{t+1}\right)\right]^{T}, \\
k=0.1, \quad \beta=0.1 .
\end{gathered}
$$

The switching signals are produced randomly with switching interval 0.2 seconds.

Remark 9. Figure 1 depicts the time response of system reference input variable $r(t)$, and Figure 2 depicts the time response of switching signals. $s w_{1}$ denotes the switching 


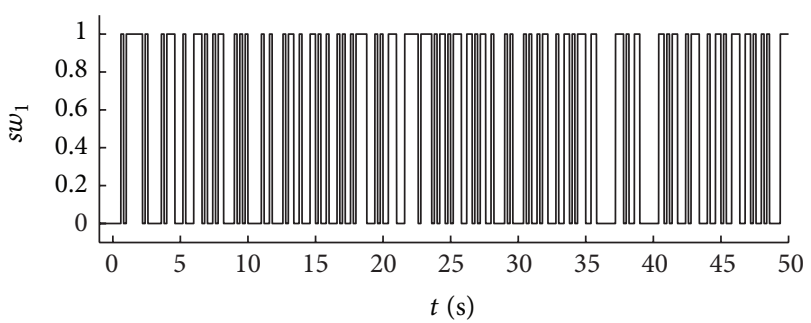

(a)

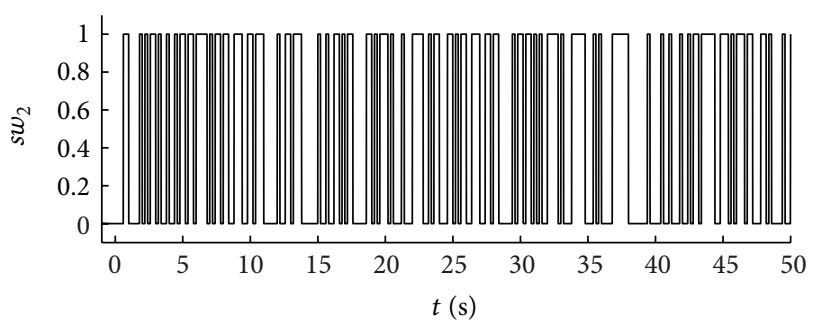

(b)

FIgURE 2: Time response of the switching variables $s w_{1}$ and $s w_{2}$.

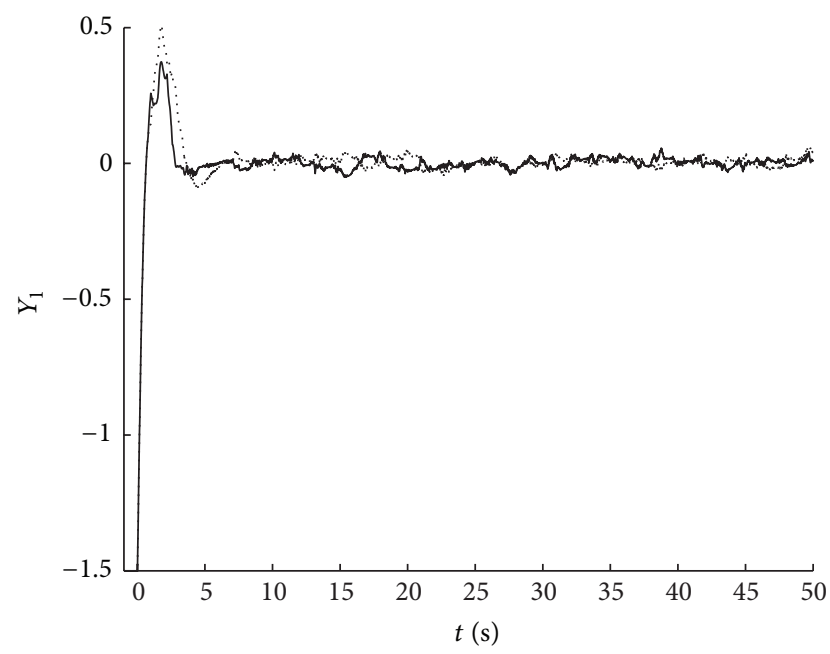

FIgURE 3: Time response of the output variable $Y_{1}(t)$.

TABLE 1: The maximal allowable delay for different parameters $k$ when $\beta$ is 0.1 .

\begin{tabular}{lccc}
\hline$k=0$ & $k=0.1$ & $k=0.2$ & $k=0.5$ \\
\hline$h_{\text {max }}=3.3395$ & $h_{\text {max }}=2.5482$ & $h_{\text {max }}=2.1313$ & $h_{\max }=1.5197$ \\
\hline
\end{tabular}

TABLE 2: The maximal allowable delay for different nonlinear parameters $\beta$ when $k$ is 0.1 .

\begin{tabular}{lccc}
\hline$\beta=0$ & $\beta=0.1$ & $\beta=0.2$ & $\beta=0.5$ \\
\hline$h_{\max }=2.6608$ & $h_{\max }=2.5482$ & $h_{\max }=2.4370$ & $h_{\max }=2.1385$ \\
\hline
\end{tabular}

signal added to the system for the first time, and $s w_{2}$ denotes the switching signal added to the system for the second time; Figure 3 depicts the time response of system output variable $Y_{1}(t)$, and Figure 4 depicts the time response of system output

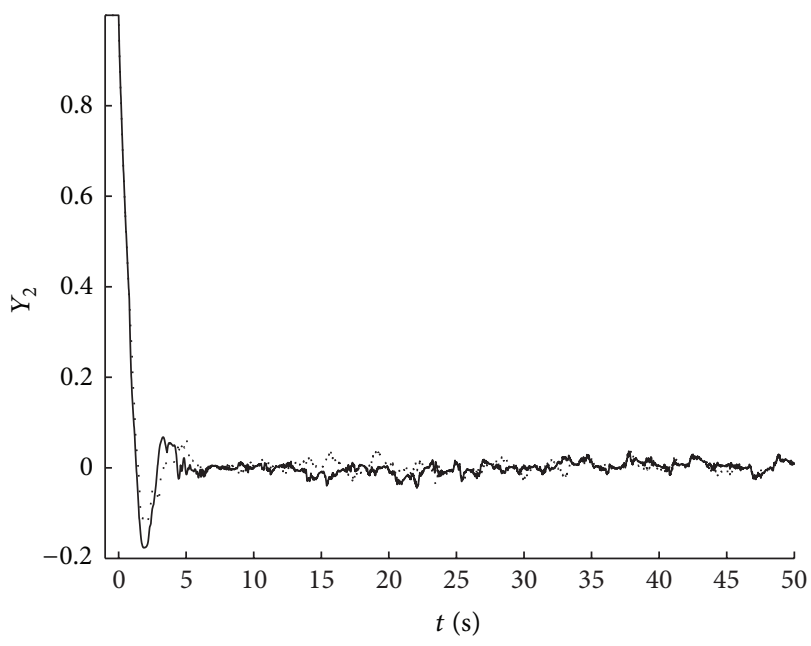

FIgURE 4: Time response of the output variable $Y_{2}(t)$.

variable $Y_{2}(t)$. The solid line denotes the output variable of the switched system with the switching signal $s w_{1}$, and the dashed line denotes the output variable of the switched system with the switching signal $s w_{2}$. From the figures it can be seen that the system output jitters in a range with a given bounded input after a period of time, which means that the system is BIBO stable and demonstrates the effectiveness of our theoretical results.

\section{Conclusions}

We have studied bounded-input bounded-output stability for a class of delay switched systems with nonlinear perturbation. Based on the Lyapunov-Krasovskii functional theory, new BIBO stabilization criteria were established in terms of delaydependent linear matrix inequalities. Some numerical simulations have been conducted to demonstrate the effectiveness of the theoretical results obtained in this paper. Future work will investigate fault detection for delay switched systems.

\section{Acknowledgments}

This work was partially supported by the Key Projects of Xihua University (Z1120946), the National Key Basic Research Program, China (2012CB215202), the 111 Project (B12018), and the National Natural Science Foundation of China (61174058 and 61134001).

\section{References}

[1] V. Kolmanovskiu and A. Myshkis, Applied Theory of FunctionalDifferential Equations, vol. 85, Kluwer Academic, Dordrecht, The Netherlands, 1992.

[2] X. Zhang, S. Li, and H. Li, "Structure and BIBO stability of a three-dimensional fuzzy two-term control system," Mathematics and Computers in Simulation, vol. 80, no. 10, pp. 1985-2004, 2010. 
[3] P. Li and S.-M. Zhong, "BIBO stabilization for system with multiple mixed delays and nonlinear perturbations," Applied Mathematics and Computation, vol. 196, no. 1, pp. 207-213, 2008.

[4] P. Li, S.-M. Zhong, and J.-Z. Cui, "Delay-dependent robust BIBO stabilization of uncertain system via LMI approach," Chaos, Solitons and Fractals, vol. 40, no. 2, pp. 1021-1028, 2009.

[5] E. Awwad, I. Győri, and F. Hartung, "BIBO stabilization of feedback control systems with time dependent delays," Applied Mathematics and Computation, vol. 219, no. 8, pp. 3664-3676, 2012.

[6] M. Mahmoud and P. Shi, "Asynchronous $H_{\infty}$ filtering of discrete-time switched systems," Signal Processing, vol. 92, no. 10, pp. 2356-2364, 2012.

[7] X. Zhao, L. Zhang, P. Shi, and M. Liu, "Stability of switched positive linear systems with average dwell time switching," Automatica, vol. 48, no. 6, pp. 1132-1137, 2012.

[8] X. Zhao, L. Zhang, P. Shi, and M. Liu, "Stability and stabilization of switched linear systems with mode-dependent average dwell time," IEEE Transactions on Automatic Control, vol. 57, no. 7, pp. 1809-1815, 2012.

[9] D. Du, B. Jiang, and P. Shi, "Sensor fault estimation and compensation for time-delay switched systems," International Journal of Systems Science, vol. 43, no. 4, pp. 629-640, 2012.

[10] Z. Wu, P. Shi, H. Su, and J. Chu, "Delay-dependent stability analysis for switched neural networks with time-varying delay," IEEE Transactions on Systems, Man, and Cybernetics B, vol. 41, no. 6, pp. 1522-1530, 2011.

[11] J. Lian, Z. Feng, and P. Shi, "Observer design for switched recurrent neural networks: an average dwell time approach," IEEE Transactions on Neural Networks, vol. 22, no. 10, pp. 15471556, 2011.

[12] D. Wang, W. Wang, and P. Shi, "Delay-dependent model reduction for continuous-time switched state-delayed systems," International Journal of Adaptive Control and Signal Processing, vol. 25, no. 9, pp. 843-854, 2011.

[13] D. Du, B. Jiang, and P. Shi, "Fault estimation and accommodation for switched systems with time-varying delay," International Journal of Control, Automation and Systems, vol. 9, no. 3, pp. 442-451, 2011.

[14] C. K. Ahn and M. K. Song, " $L_{2}-L_{\infty}$ filtering for time-delayed switched hopfield neural networks," International Journal of Innovative Computing, Information and Control, vol. 7, no. 4, pp. 1831-1843, 2011.

[15] Z. G. Wu, P. Shi, H. Su, and J. Chu, "Delay-dependent exponential stability analysis for discrete-time switched neural networks with time-varying delay," Neurocomputing, vol. 74, no. 10, pp. 1626-1631, 2011.

[16] G. Zong, L. Hou, and J. Li, "A descriptor system approach to $L$ ${ }_{2}-L_{\infty}$ filtering for uncertain discrete-time switched system with mode-dependent time-varying delays," International Journal of Innovative Computing, Information and Control, vol. 7, no. 5A, pp. 2213-2224, 2011.

[17] Y. Wang, W. Wang, and D. Wang, "LMI approach to design fault detection filter for discrete-time switched systems with state delays," International Journal of Innovative Computing, Information and Control, vol. 6, no. 1, pp. 387-397, 2010.

[18] L. Zhang and B. Jiang, "Stability of a class of switched linear systems with uncertainties and average dwell time switching," International Journal of Innovative Computing, Information and Control, vol. 6, no. 2, pp. 667-676, 2010.
[19] F. Long, C. Li, and C. Cui, "Dynamic output feedback $H_{\infty}$ control for a class of switched linear systems with exponential uncertainty," International Journal of Innovative Computing, Information and Control, vol. 6, no. 4, pp. 1727-1736, 2010.

[20] H. R. Karimi, "Robust synchronization and fault detection of uncertain master-slave systems with mixed time-varying delays and nonlinear perturbations," International Journal of Control, Automation and Systems, vol. 9, no. 4, pp. 671-680, 2011.

[21] H. R. Karimi, "Adaptive $H_{\infty}$ synchronization problem of uncertain master-slave systems with mixed time-varying delays and nonlinear perturbations: an LMI approach," International Journal of Automation and Computing, vol. 8, no. 4, pp. 381-390, 2011.

[22] P. Shi, X. Luan, and F. Liu, " $H_{\infty}$ filtering for discrete-time systems with stochastic incomplete measurement and mixed delays," IEEE Transactions on Industrial Electronics, vol. 59, pp. 2732-2739, 2012.

[23] H. R. Karimi, M. Zapateiro, and N. Luo, "Stability analysis and control synthesis of neutral systems with time-varying delays and nonlinear uncertainties," Chaos, Solitons and Fractals, vol. 42, no. 1, pp. 595-603, 2009.

[24] M. Basin, P. Shi, and P. Soto, "Central suboptimal $H_{\infty}$ filtering for nonlinear polynomial systems with multiplicative noise," Journal of the Franklin Institute, vol. 347, no. 9, pp. 1740-1754, 2010.

[25] H. R. Karimi, N. Luo, M. Zapateiro, and L. Zhang, " $H_{\infty}$ control design for building structures under seismic motion with wireless communication," International Journal of Innovative Computing, Information and Control, vol. 7, pp. 5269-5284, 2011.

[26] H. R. Karimi, "A sliding mode approach to $H_{\infty}$ synchronization of master-slave time-delay systems with Markovian jumping parameters and nonlinear uncertainties," Journal of the Franklin Institute. Engineering and Applied Mathematics, vol. 349, no. 4, pp. 1480-1496, 2012.

[27] X. Li and H. Gao, "A new model transformation of discrete-time systems with time-varying delay and its application to stability analysis," IEEE Transactions on Automatic Control, vol. 56, no. 9, pp. 2172-2178, 2011.

[28] X. Li, H. Gao, and X. Yu, "A unified approach to the stability of generalized static neural networks with linear fractional uncertainties and delays," IEEE Transactions on System, Man, and Cybernetics B, vol. 41, no. 5, pp. 1275-1286, 2011.

[29] H. Gao and X. Li, " $H_{\infty}$ filtering for discrete-time state-delayed systems with finite frequency specifications," IEEE Transactions on Automatic Control, vol. 56, no. 12, pp. 2935-2941, 2011.

[30] S. Boyd, L. El Ghaoui, E. Feron, and V. Balakrishnan, Linear Matrix Inequalities in System and Control Theory, vol. 15, Society for Industrial and Applied Mathematics, Philadelphia, Pa, USA, 1994.

[31] K. Gu, "An integral inequality in the stability problem of time-delay systems," in Proceedings of the 39th IEEE Confernce on Decision and Control, pp. 2805-2810, Sydney, Australia, December 2000

[32] H. S. Wu and K. Mizukami, "Robust stabilization of uncertain linear dynamical systems," International Journal of Systems Science, vol. 24, no. 2, pp. 265-276, 1993. 


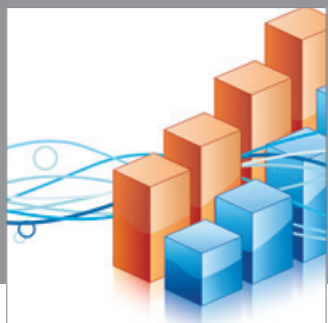

Advances in

Operations Research

mansans

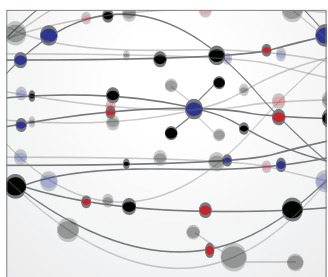

The Scientific World Journal
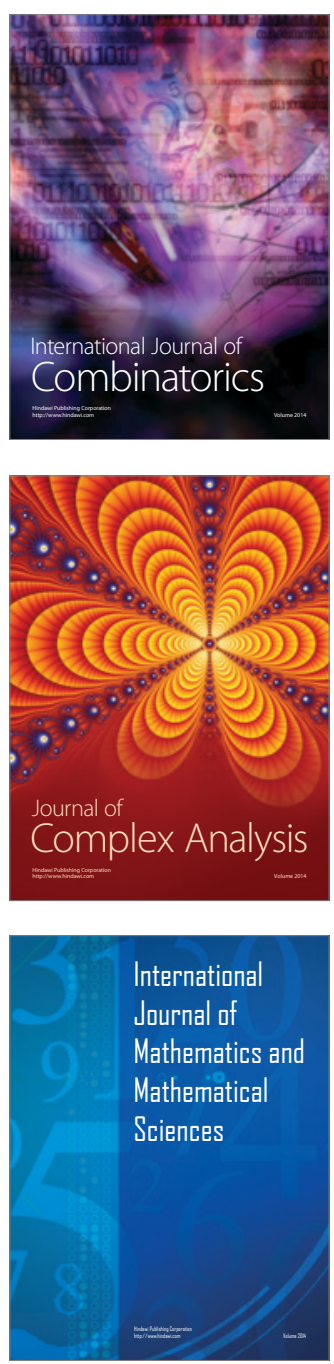
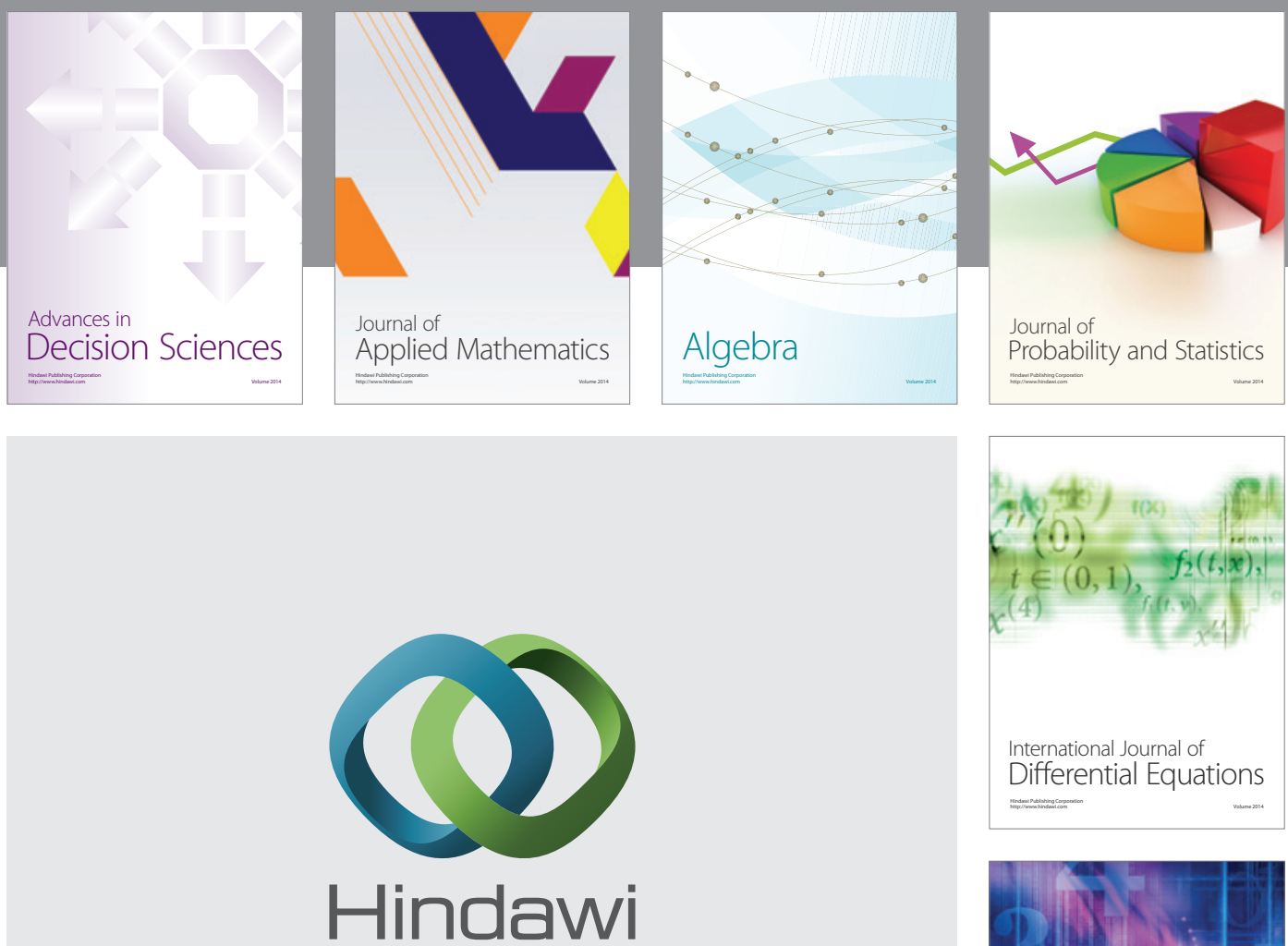

Submit your manuscripts at http://www.hindawi.com
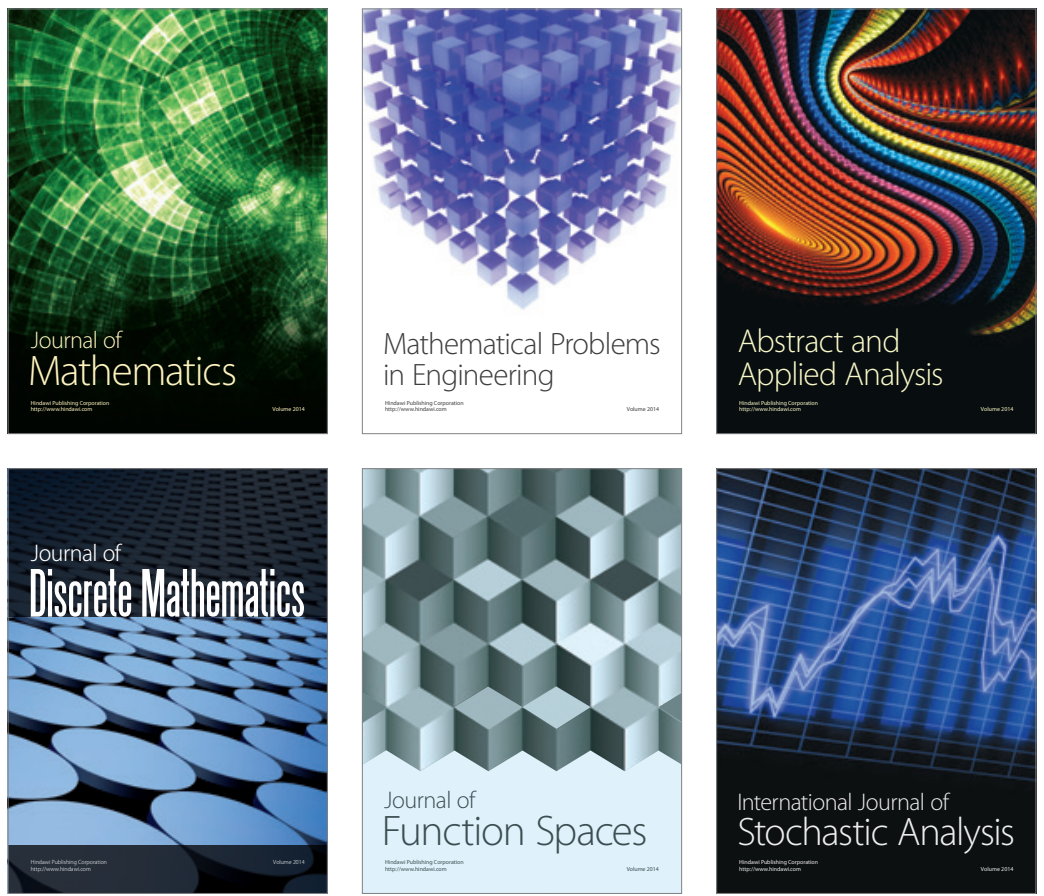

Journal of

Function Spaces

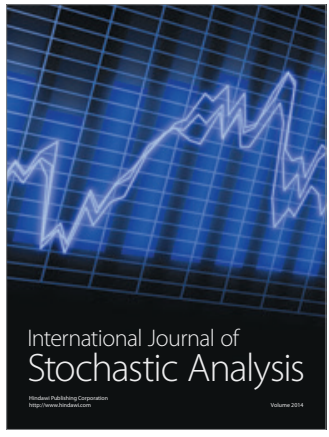

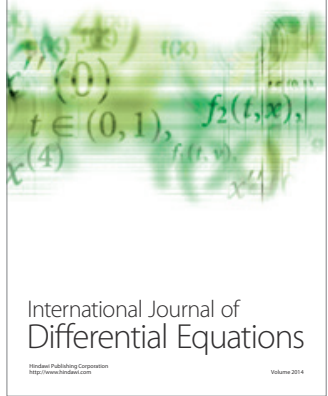
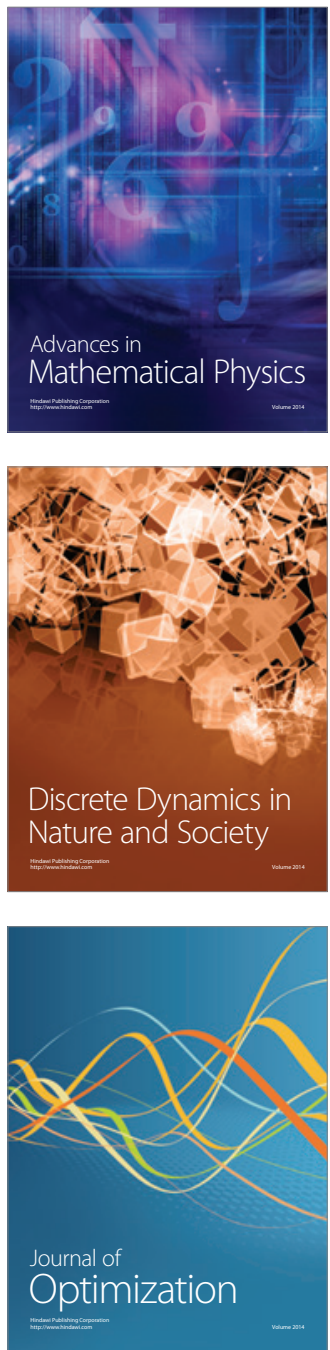